Modeling, Identification and Control, Vol. 29, No. 2, 2008, pp. 51-58

\title{
Observer Design for Second-Order Distributed Parameter Systems in $\mathbb{R}^{2}$
}

\author{
Tu Duc Nguyen ${ }^{1}$ \\ ${ }^{1}$ Department of Engineering Cybernetics, Norwegian University of Science and Technology, N-7491 Trondheim, \\ Norway. E-mail: Tu.Duc.Nguyen@itk.ntnu.no
}

\begin{abstract}
Observer design for second-order distributed parameter systems in $\mathbb{R}^{2}$ is addressed. Particularly, second order distributed parameter systems without distributed damping are studied. Based on finite number of measurements, exponentially stable observer is designed. The existence, uniqueness and stability of solutions of the observer are based on semigroup theory.
\end{abstract}

Keywords: Distributed parameter systems; Observers; Second-order systems; Semigroup.

\section{Introduction}

Observer design for dynamic systems has been extensively studied by numerous of authors (see e.g. Anderson and Moore (1990), Balas (1999), Gauthier and Kupka (2001),Luenberger (1979),Nijmeijer and (eds.) and references therein). Most of these observers were mainly developed for dynamic systems described by ordinary differential equations (ODEs), i.e. finitedimensional models. There exist few results for dynamic systems described by partial differential equations (PDEs), i.e. infinite-dimensional models. Particularly, for second order distributed parameter systems. Traditionally, observers for infinite-dimensional model are designed via finite dimensional models, i.e. some finite-dimensional approximation scheme is applied to the infinite-dimensional model, e.g. finite element method, finite difference method, finite volume method, etc., and a set of $n$-2nd order ODEs is obtained. These $n$-2nd order ODEs are then converted into a vector first order form, and observers for the original system are then designed after this step. The main drawback of this approach is the loss of the advantageous algebraic structure of the second-order systems. Additionally, this approach does not ensure that the estimated states are the estimates of the true state. This is argued in great details in Balas (1999),Demetriou (2004).

In Smyshlyaev and Kristic (2005), Smyshlyaev and Krstic considered backstepping observers for a class of parabolic PDEs. Vazquez and Krstic Vazquez and Krstic (2005) presented a nonlinear PDE observer for the channel flow Navier-Stokes system. Bounit and Hammouri Bounit and Hammouri (1997) studied observer design for infinite dimensional bilinear systems, described by vector first order systems. Balas Balas (1999) considered observer design for linear flexible structures described by FEM. Recently, Xu et al. Xu and Sallet (2006) considered infinite dimensional observers for vibrating systems. Kalman type observers were proposed, and it was shown that the observer error system might become unstable for large observer gain. In (Demetriou (2004), Kristiansen (2000)), a method for construction of observer for linear second order distributed parameter systems is presented. The damping forces were included in both cases. Thus, exponentially stable observers can easily be designed. In (Nguyen and Egeland (2003), Nguyen and Egeland (2006)), as opposed to the work of (Demetriou (2004),Kristiansen (2000)), observer design for one dimensional second order distributed parameter systems without strictly positive damping is studied. This note 
extends the approach in Nguyen and Egeland (2006) to two dimensional second-order distributed parameter systems. The main analysis tool is the semigroup theory.

The paper is organized as follows. First, a model of the system is presented. Then, observer design is studied. Finally, concluding remarks are given.

\section{System Model}

We consider dynamic systems of the form

$$
\rho w_{t t}+\mathcal{C} w_{t}+\mathcal{K} w=\mathcal{B} u, \quad(\mathbf{x}, t) \in \Omega \times \mathbb{R}^{+}
$$

where

$$
\begin{aligned}
\mathcal{K} w & =\sum_{i=1}^{2}(-1)^{i} \nabla^{i}\left(k_{i} \nabla^{i} w\right) \\
\mathcal{C} w_{t} & =c_{0} w_{t}+\sum_{i=1}^{2}(-1)^{i} \nabla^{i}\left(c_{i} \nabla^{i} w_{t}\right)
\end{aligned}
$$

$\mathcal{K}$ is the stiffness operator of the system with stiffness coefficients $k_{i}>0, \mathcal{C}$ denotes the damping operator of the system with damping coefficients $c_{i} \geq 0, \Omega \subset \mathbb{R}^{2}$ is the domain of definition, $\rho$ represents the mass density of the flexible structure, $w(\mathbf{x}, t) \in H_{0}^{2 p}(\Omega) \subset H^{2 p}(\Omega)$ is the vertical displacement of the structure at $\mathbf{x} \in \Omega$ and time $t \geq 0, u(\mathbf{x}, t) \in \mathcal{U}$ is the control signal generated at $\mathbf{x} \in \Omega$ and time $t \geq 0, \mathcal{B}: \mathcal{U} \rightarrow L_{2}(\Omega)$ is the input operator (typically of type $\mathcal{L}\left(\mathcal{U}, L_{2}(\Omega)\right.$ ), i.e. a bounded linear operator that maps from $\mathcal{U}$ to $\left.L_{2}(\Omega)\right), \mathcal{U}$ is the space of input signals, and the standard spaces $L_{2}(\Omega), H^{m}(\Omega)$ and $H_{0}^{m}(\Omega)$ are for the sake of clarity given below. The subscript $(\cdot)_{t}$ denotes the partial differential with respect to $t$. This notation will be applied throughout the paper.

The boundary conditions associated with (1) are given as

$$
\begin{aligned}
w & =0, \Gamma_{0} \times \mathbb{R}^{+}(2) \\
k_{1} \nabla w \cdot \mathbf{n} & =0, \Gamma_{0} \times \mathbb{R}^{+}(3) \\
k_{2} \nabla^{2} w & =0, \Gamma_{1} \times \mathbb{R}^{+}(4) \\
\sum_{i=1}^{2}(-1)^{i-1} \nabla^{i-1}\left(k_{i} \nabla^{i} w\right) \cdot \mathbf{n}+c_{\Gamma} w_{t} & =0, \Gamma_{1} \times \mathbb{R}^{+}(5)
\end{aligned}
$$

where $c_{\Gamma}>0$ is the boundary damping coefficient, $\Gamma_{i}$ are the boundaries of the domain $\Omega$, and $\mathbf{n}$ is the unitnormal vector of $\Gamma_{i}$ pointing outward of $\Omega$.

The initial conditions of (1)-(5) are denoted as

$$
\begin{aligned}
w(\mathbf{x}, 0) & =W_{0}(\mathbf{x}), \bar{\Omega} \\
w_{t}(\mathbf{x}, 0) & =V_{0}(\mathbf{x}), \bar{\Omega}
\end{aligned}
$$

where $\bar{\Omega}=\Omega \cup \Gamma, \Gamma=\Gamma_{0} \cup \Gamma_{1}, W_{0}$ and $V_{0}$ are the initial position and velocity functions of the flexible structure, respectively.
Note that with $p=1$ and $p=2$, we get the damped membrane equation and plate equation, respectively,

$$
\begin{aligned}
\rho w_{t t} & =-\mathcal{C} w_{t}+\nabla\left(k_{1} \nabla w\right)+\mathcal{B} u, \quad \Omega \times \mathbb{R}^{+} \\
\rho w_{t t} & =-\mathcal{C} w_{t}-\nabla^{2}\left(k_{2} \nabla^{2} w\right)+\nabla\left(k_{1} \nabla w\right)+\mathcal{B} u, \quad \Omega \times \mathbb{R}^{+}
\end{aligned}
$$

\subsection{Basic Equalities, Inequalities and Spaces}

The most frequently used equalities, inequalities and spaces in this note are collected here.

Let $\Omega$ be a bounded domain of $\mathbb{R}^{n}$ with smooth boundary $\Gamma$. We denote the space of all equivalence classes of real-valued Lebesgue-measurable functions by $L_{p}(\Omega), 1 \leq p \leq \infty$. $L_{p}(\Omega)$ is a Banach space with the norm

$$
\begin{aligned}
& \|w\|_{L_{p}(\Omega)}=\left(\int_{\Omega}|w|^{p} d \Omega\right)^{\frac{1}{p}}, \quad 1 \leq p<\infty \\
& \|w\|_{L_{\infty}(\Omega)}=\quad \operatorname{ess} \sup _{\mathbf{x} \in \Omega}|w(\mathbf{x})|, \quad p=\infty
\end{aligned}
$$

For $m \in \mathbb{N}, 1 \leq p \leq \infty, W^{m, p}(\Omega)$ is defined to be the Sobolev space consisting of all functions $w$ in $L_{p}(\Omega)$ whose distribution derivatives of order up to $m$ are also in $L_{p}(\Omega)$. It is known that $W^{m, p}(\Omega)$ is a Banach space with the norm

$$
\|w\|_{W^{m, p}(\Omega)}=\left(\sum_{|\alpha| \leq m}\left\|D^{\alpha} w\right\|_{L_{p}(\Omega)}^{p}\right)^{\frac{1}{p}}
$$

where $\alpha=\left\{\alpha_{1}, \ldots, \alpha_{n}\right\} \in \mathbb{N}^{n},|\alpha|=\alpha_{1}+\ldots+\alpha_{n}$, $D^{\alpha} w=\frac{\partial^{\alpha_{1}+\cdots+\alpha_{n}} w}{\partial x_{1}^{\alpha_{1}} \cdots \partial x_{n}^{\alpha_{n}}}$. When $p=2$, the space $W^{m, p}(\Omega)$ is denoted by $H^{m}(\Omega)$. It is well-known that the Sobolev space $H^{m}(\Omega)$ is a Hilbert space with the corresponding inner product.

Now, let $\Omega$ be a bounded domain of $\mathbb{R}^{2}$ and $m \in$ $\{2,3,4, \ldots\}$. Consider the subspace of $H^{m}(\Omega)$ defined as

$$
\begin{aligned}
H_{0}^{m}(\Omega)= & \left\{f \mid f, \nabla f, \ldots, \nabla^{m} f \in L_{2}(\Omega),\right. \\
& \left.\left.f\right|_{\Gamma_{0}}=\left.\nabla f \cdot \mathbf{n}\right|_{\Gamma_{0}}=0\right\}
\end{aligned}
$$

where $\mathbf{n}$ is the unit-normal vector to $\Gamma$ pointing outward of $\Omega$, and

$$
L_{2}(\Omega)=\left\{f \mid\left(\int_{\Omega}|f(x)|^{2} d x\right)^{\frac{1}{2}}<\infty\right\}
$$

We have the Poincare inequality and Sobolev inequality

$$
\begin{aligned}
& \int_{\Omega}|f|^{2} d x \leq \mathrm{c}_{1} \int_{\Omega}\left|\nabla^{j} f\right|^{2} d x \\
& \int_{\Gamma_{1}}|f|^{2} d \Gamma \leq \mathrm{c}_{2} \int_{\Omega}\left|\nabla^{j} f\right|^{2} d x
\end{aligned}
$$


for $j=1,2, \forall f \in H_{0}^{m}(\Omega)$, and some constants $\mathrm{c}_{1}, \mathrm{c}_{2}>$ 0 .

The normal derivative of $f(x)$ is defined as

$$
\frac{\partial f}{\partial n}=\nabla f \cdot \mathbf{n}, \quad \Gamma
$$

where $\mathbf{n}$ is the unit-normal vector to $\Gamma$ pointing outward of $\Omega$. For $f \in H^{1}(\Omega)$ and $g \in H^{2}(\Omega)$, we have the integral equalities

$$
\begin{aligned}
\int_{\Omega} \nabla^{2} g f d x & =\int_{\Gamma} \nabla g \cdot \mathbf{n} f d \Gamma-\int_{\Omega} \nabla g \cdot \nabla f d x \\
\int_{\Omega} \mathbf{r} \cdot \nabla f d x & =\int_{\Gamma} \mathbf{r} \cdot \mathbf{n} f d \Gamma-\int_{\Omega} \nabla \cdot \mathbf{r} f d x
\end{aligned}
$$

Let $\mathbf{a}, \mathbf{b} \in \mathbb{R}^{2}$, we have

$$
\mathbf{a} \cdot \mathbf{b} \leq(\mu|\mathbf{a}|)^{2}+\left(\frac{|\mathbf{b}|}{\mu}\right)^{2}, \quad \mu \in \mathbb{R} \backslash\{0\}
$$

\subsection{Assumptions}

We assume

A.1 $\rho>0, k_{i}>0, c_{i} \geq 0, c_{\Gamma}>0$ are constant, and

$$
c_{i}=\alpha k_{i}, \quad i=1,2
$$

for some constant $\alpha \geq 0$.

A.2 $\Omega$ is rectangular or formed by smooth boundary $\Gamma$ such that the following holds

$$
\begin{aligned}
& \mathbf{r} \cdot \mathbf{n} \leq 0, \quad \Gamma_{0} \\
& \mathbf{r} \cdot \mathbf{n}>0, \quad \Gamma_{1}
\end{aligned}
$$

where $\mathbf{r}=\mathbf{x}$ and $\mathbf{n}$ is the unit-normal vector of $\Gamma_{i}$ pointing outward of $\Omega$.

\subsection{Problem Statement}

This note addresses the problem:

Problem 1: Given the system (1)-(5) and measurements,

$$
y_{i}(\mathbf{x}, t)=w_{t} \cdot \chi_{i}(\mathbf{x}), \quad \mathbf{x} \in \Omega_{i}, t \geq 0
$$

for $i=1,2, \ldots, N$, where $\left.\Omega_{i}=\right] x_{1, i}-\epsilon_{i}, x_{1, i}+\epsilon_{i}[\times$ ]$x_{2, i}-\epsilon_{i}, x_{2, i}+\epsilon_{i}\left[\subset \Omega, \epsilon_{i}>0\right.$ are small positive constants, and $\chi_{i}: \Omega_{i} \rightarrow \mathbb{R}^{+}$are given smooth distribution functions (Figure 1). Design an observer for the system (1)-(5).

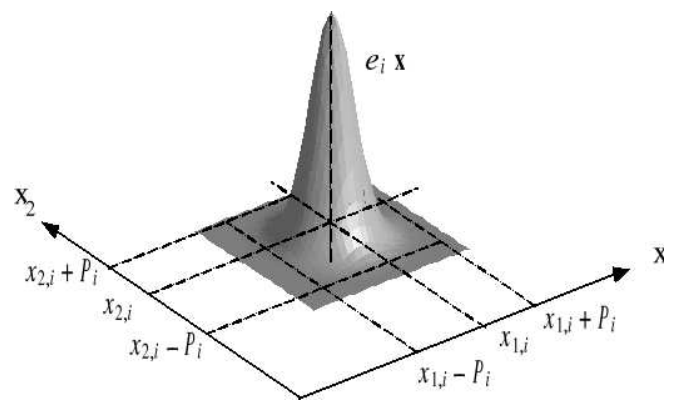

Figure 1: Distribution function $\chi_{i}$

\section{Observer Design}

Copying the model (1)-(7) and adding output injection terms, we get the observer

$$
\begin{aligned}
\rho \hat{w}_{t t}= & -\mathcal{C} \hat{w}_{t}-\mathcal{K} \hat{w}+\mathcal{B} u \\
& -\sum_{i=1}^{N} H_{i} \cdot\left(\hat{w}_{t}-y_{i}\right) \cdot \chi_{i}, \quad \Omega \times \mathbb{R}^{+}
\end{aligned}
$$

with the boundary conditions

$$
\begin{aligned}
\hat{w} & =0, \Gamma_{0} \times \mathbb{R}^{+}(17) \\
k_{1} \nabla \hat{w} \cdot \mathbf{n} & =0, \Gamma_{0} \times \mathbb{R}^{+}(18) \\
k_{2} \nabla^{2} \hat{w} & =0, \Gamma_{1} \times \mathbb{R}^{+}(19) \\
\sum_{i=1}^{2}(-1)^{i-1} \nabla^{i-1}\left(k_{i} \nabla^{i} \hat{w}\right) \cdot \mathbf{n}+c_{\Gamma} \hat{w}_{t} & =0, \Gamma_{1} \times \mathbb{R}^{+}(20)
\end{aligned}
$$

and initial conditions

$$
\begin{aligned}
\hat{w}(\mathbf{x}, 0) & =\hat{W}_{0}(\mathbf{x}), \quad \bar{\Omega} \\
\hat{w}_{t}(\mathbf{x}, 0) & =\hat{V}_{0}(\mathbf{x}), \quad \bar{\Omega}
\end{aligned}
$$

where $\hat{w}$ denotes the estimate of $w, H_{i}>0$ are the observer gains, and $\hat{W}_{0}$ and $\hat{V}_{0}$ denote the initial position and velocity functions of the observer, respectively.

Subtracting (16)-(22) by (1)-(7) gives the error dynamics

$$
\rho \tilde{w}_{t t}=-\mathcal{C} \tilde{w}_{t}-\mathcal{K} \tilde{w}-\sum_{i=1}^{N} H_{i} \tilde{w}_{t} \chi_{i}, \quad \Omega \times \mathbb{R}^{+}
$$

with the boundary conditions

$$
\begin{aligned}
\tilde{w} & =0, \Gamma_{0} \times \mathbb{R}^{+}(24) \\
k_{1} \nabla \tilde{w} \cdot \mathbf{n} & =0, \Gamma_{0} \times \mathbb{R}^{+}(25) \\
k_{2} \nabla^{2} \tilde{w} & =0, \Gamma_{1} \times \mathbb{R}^{+}(26) \\
\sum_{i=1}^{2}(-1)^{i-1} \nabla^{i-1}\left(k_{i} \nabla^{i} \tilde{w}\right) \cdot \mathbf{n}+c_{\Gamma} \tilde{w}_{t} & =0, \Gamma_{1} \times \mathbb{R}^{+}(27)
\end{aligned}
$$

and initial conditions

$$
\begin{aligned}
\tilde{w}(\mathbf{x}, 0) & =\hat{W}_{0}-W_{0}, \quad \mathbf{x} \in \bar{\Omega} \\
\tilde{w}_{t}(\mathbf{x}, 0) & =\hat{V}_{0}-V_{0}, \quad \mathbf{x} \in \bar{\Omega}
\end{aligned}
$$


where $\tilde{w}=\hat{w}-w$ denotes the observer error.

Now, we divide the stability analysis of (23)-(29) into two parts. First, the case where $c_{0}, \alpha>0$, i.e. strictly positive distributed damping, is studied. A Lyapunovlike argumentation will be applied.

The second part treats the case where $c_{0}, \alpha \geq 0$. To show the well-posedness and stability of the observer (16)-(22), the semigroup theory in combination with the energy-multiplier method are applied. This is the main contribution of the note.

\subsection{Case 1: Strictly Positive Distributed Damping}

Let the damping coefficients be strictly positive, i.e. $c_{i}>0$. Consider the Lyapunov functional

$$
\mathcal{W}\left(t, \tilde{w}, \tilde{w}_{t}\right)=\mathcal{E}+\gamma \int_{\Omega} \rho \tilde{w}_{t} \tilde{w} d x
$$

where $\mathcal{E}$ is the natural energy functional given as

$$
\begin{aligned}
\mathcal{E}= & \frac{1}{2} \int_{\Omega} \rho \tilde{w}_{t}^{2} d x+\frac{1}{2} \int_{\Omega} \sum_{i=1}^{2} k_{i}\left|\nabla^{i} \tilde{w}\right|^{2} d x \\
& +\frac{1}{2} \int_{\Omega} 2(1-\nu)\left[\left(\frac{\partial^{2} \tilde{w}}{\partial x_{1} \partial x_{2}}\right)^{2}-\frac{\partial^{2} \tilde{w}}{\partial x_{1}^{2}} \frac{\partial^{2} \tilde{w}}{\partial x_{2}^{2}}\right] d x(31)
\end{aligned}
$$

$\nu>0$ denotes the Poisson's ratio, and $\gamma>0$ is the Lyapunov gain (to be determined below). The first term and the last two terms represent the kinetic energy and potential energy of the observer error dynamics (23)-(27), respectively. Due to the boundary conditions (24)-(27) and the assumption A.2, the Gaussian curvature integral is zero I. and Dym (1991).

Neglecting the Gaussian curvature integral, and application of (10) to (31) yields

$$
\begin{aligned}
\mathcal{W} \geq & \frac{1}{2} \int_{\Omega} \rho \tilde{w}_{t}^{2} d x+\gamma \int_{\Omega} \rho \tilde{w}_{t} \tilde{w} d x \\
& +\frac{1}{2} \int_{\Omega} \frac{k_{1}}{c_{1}}|\tilde{w}|^{2} d x+\frac{1}{2} \int_{\Omega} k_{2}\left|\nabla^{2} \tilde{w}\right|^{2} d x
\end{aligned}
$$

for some constant $c_{1}>0$. Choosing

$$
|\gamma|<\sqrt{\frac{k_{1}}{\rho \mathbf{c}_{1}}}
$$

ensures that

$$
\mathcal{W}>0, \quad \forall\left(\tilde{w}, \tilde{w}_{t}\right) \neq 0
$$

Assume now that the initial conditions (28)-(29) are sufficiently smooth such that the problem (23)-(29) is well-posed (see next sub-section and Remark 2). Taking the time derivative of (30) along the solution tra- jectories of (23)-(27) gives

$$
\begin{aligned}
\dot{\mathcal{W}}= & \sum_{i=1}^{10} \dot{\mathcal{W}}_{i} \\
= & -\int_{\Omega} c_{0} \tilde{w}_{t}^{2} d x-\int_{\Omega} \sum_{j=1}^{N} H_{j} \chi_{j} \tilde{w}_{t}^{2} d x \\
& -\int_{\Omega} \tilde{w}_{t} \sum_{i=1}^{2}(-1)^{i} \nabla^{i}\left(c_{i} \nabla^{i} \tilde{w}_{t}\right) d x \\
& -\int_{\Omega} \tilde{w}_{t} \sum_{i=1}^{2}(-1)^{i} \nabla^{i}\left(k_{i} \nabla^{i} \tilde{w}\right) d x \\
& +\int_{\Omega}\left[k_{1} \nabla \tilde{w} \cdot \nabla \tilde{w}_{t}+k_{2} \nabla^{2} \tilde{w} \nabla^{2} \tilde{w}_{t}\right] d x \\
& +\gamma \int_{\Omega} \rho \tilde{w}_{t}^{2} d x-\gamma \int_{\Omega} c_{0} \tilde{w} \tilde{w}_{t} d x \\
& -\gamma \int_{\Omega} \tilde{w} \sum_{i=1}^{2}(-1)^{i} \nabla^{i}\left(c_{i} \nabla^{i} \tilde{w}_{t}\right) d x \\
& -\gamma \int_{\Omega} \tilde{w} \sum_{j=1}^{N} H_{j} \chi_{j} \tilde{w}_{t} d x \\
& -\gamma \int_{\Omega} \tilde{w} \sum_{i=1}^{2}(-1)^{i} \nabla^{i}\left(k_{i} \nabla^{i} \tilde{w}\right) d x
\end{aligned}
$$

Application of (13)-(14) and (24)-(27) to $\dot{\mathcal{W}}_{3}, \dot{\mathcal{W}}_{4}, \dot{\mathcal{W}}_{8}$ and $\dot{\mathcal{W}}_{10}$ give

$$
\begin{aligned}
\dot{\mathcal{W}}_{3}= & -\int_{\Omega} \tilde{w}_{t} \sum_{i=1}^{2}(-1)^{i} \nabla^{i}\left(c_{i} \nabla^{i} \tilde{w}_{t}\right) d x \\
= & \int_{\Gamma} \tilde{w}_{t} c_{1} \nabla \tilde{w}_{t} \cdot \mathbf{n} d \Gamma-\int_{\Omega}\left[c_{1}\left|\nabla \tilde{w}_{t}\right|^{2}+c_{2}\left|\nabla^{2} \tilde{w}_{t}\right|^{2}\right] d x \\
& -\int_{\Gamma} \tilde{w}_{t} \nabla\left(c_{2} \nabla^{2} \tilde{w}_{t}\right) \cdot \mathbf{n} d \Gamma+\int_{\Gamma} c_{2} \nabla^{2} \tilde{w}_{t} \nabla \tilde{w}_{t} \cdot \mathbf{n} d \Gamma \\
= & -\int_{\Gamma_{1}} \alpha c_{\Gamma} \tilde{w}_{t}^{2} d \Gamma-\int_{\Omega}\left[c_{1}\left|\nabla \tilde{w}_{t}\right|^{2}+c_{2}\left|\nabla^{2} \tilde{w}_{t}\right|^{2}\right] d x \\
\dot{\mathcal{W}}_{4}= & -\int_{\Omega} \tilde{w}_{t} \sum_{i=1}^{2}(-1)^{i} \nabla^{i}\left(k_{i} \nabla^{i} \tilde{w}\right) d x \\
= & \int_{\Gamma} \tilde{w}_{t} k_{1} \nabla \tilde{w}^{\prime} \mathbf{n} d \Gamma-\int_{\Omega} k_{1} \nabla \tilde{w}_{t} \cdot \nabla \tilde{w} d x \\
& -\int_{\Gamma} \tilde{w}_{t} \nabla\left(k_{2} \nabla^{2} \tilde{w}\right) \cdot \mathbf{n} d \Gamma+\int_{\Gamma} k_{2} \nabla \tilde{w}_{t} \cdot \mathbf{n} \nabla^{2} \tilde{w} d \Gamma \\
& -\int_{\Omega} k_{2} \nabla^{2} \tilde{w}_{t} \nabla^{2} \tilde{w} d x \\
= & -\int_{\Gamma_{1}} c_{\Gamma} \tilde{w}_{t}^{2} d \Gamma-\int_{\Omega}\left[k_{1} \nabla \tilde{w}_{t} \cdot \nabla \tilde{w}+k_{2} \nabla^{2} \tilde{w}_{t} \nabla^{2} \tilde{w}\right] d x
\end{aligned}
$$




$$
\begin{aligned}
\frac{\dot{\mathcal{W}}_{8}}{\gamma}= & -\int_{\Omega} \tilde{w} \sum_{i=1}^{2}(-1)^{i} \nabla^{i}\left(c_{i} \nabla^{i} \tilde{w}_{t}\right) d x \\
= & \int_{\Gamma} c_{1} \tilde{w} \nabla \tilde{w}_{t} \cdot \mathbf{n} d \Gamma-\int_{\Omega} c_{1} \nabla \tilde{w} \cdot \nabla \tilde{w}_{t} d x \\
& -\int_{\Gamma} \tilde{w} \nabla\left(c_{2} \nabla^{2} \tilde{w}_{t}\right) \cdot \mathbf{n} d \Gamma+\int_{\Gamma} c_{2} \nabla \tilde{w} \cdot \mathbf{n} \nabla^{2} \tilde{w}_{t} d \Gamma \\
& -\int_{\Omega} c_{2} \nabla^{2} \tilde{w} \nabla^{2} \tilde{w}_{t} d x \\
= & -\int_{\Gamma_{1}} \alpha c_{\Gamma} \tilde{w} \tilde{w}_{t} d \Gamma-\int_{\Omega}\left[c_{1} \nabla \tilde{w} \cdot \nabla \tilde{w}_{t}+c_{2} \nabla^{2} \tilde{w} \nabla^{2} \tilde{w}_{t}\right]
\end{aligned}
$$

$$
\begin{aligned}
\frac{\dot{\mathcal{W}}_{10}}{\gamma}= & -\int_{\Omega} \tilde{w} \sum_{i=1}^{2}(-1)^{i} \nabla^{i}\left(k_{i} \nabla^{i} \tilde{w}\right) d x \\
= & \int_{\Gamma} \tilde{w} k_{1} \nabla \tilde{w} \cdot \mathbf{n} d \Gamma-\int_{\Omega} k_{1}|\nabla \tilde{w}|^{2} d x \\
& -\int_{\Gamma} \tilde{w} \nabla\left(k_{2} \nabla^{2} \tilde{w}\right) \cdot \mathbf{n} d \Gamma+\int_{\Gamma} k_{2} \nabla^{2} \tilde{w} \nabla \tilde{w} \cdot \mathbf{n} d \Gamma \\
& -\int_{\Omega} k_{2}\left|\nabla^{2} \tilde{w}\right|^{2} d x \\
= & -\int_{\Gamma_{1}} c_{\Gamma} \tilde{w} \tilde{w}_{t} d \Gamma-\int_{\Omega}\left[k_{1}|\nabla \tilde{w}|^{2}+k_{2}\left|\nabla^{2} \tilde{w}\right|^{2}\right] d x
\end{aligned}
$$

Thus,

$$
\begin{aligned}
\dot{\mathcal{W}}= & -\int_{\Omega}\left(c_{0}-\gamma \rho\right) \tilde{w}_{t}^{2} d x-\int_{\Omega} \sum_{j=1}^{N} H_{j} \chi_{j} \tilde{w}_{t}^{2} d x \\
& -\int_{\Gamma_{1}} c_{\Gamma}(1+\alpha) \tilde{w}_{t}^{2} d \Gamma \\
& -\int_{\Omega}\left[c_{1}\left|\nabla \tilde{w}_{t}\right|^{2}+c_{2}\left|\nabla^{2} \tilde{w}_{t}\right|^{2}\right] d x \\
& -\gamma \int_{\Omega} c_{0} \tilde{w} \tilde{w}_{t} d x-\gamma \int_{\Gamma_{1}} \alpha c_{\Gamma} \tilde{w} \tilde{w}_{t} d \Gamma \\
& -\gamma \int_{\Omega}\left[c_{1} \nabla \tilde{w} \cdot \nabla \tilde{w}_{t}+c_{2} \nabla^{2} \tilde{w} \nabla^{2} \tilde{w}_{t}\right] d x \\
& -\gamma \int_{\Omega} \tilde{w} \sum_{j=1}^{N} H_{j} \chi_{j} \tilde{w}_{t} d x-\gamma \int_{\Gamma_{1}} c_{\Gamma} \tilde{w} \tilde{w}_{t} d \Gamma \\
& -\gamma \int_{\Omega}\left[k_{1}|\nabla \tilde{w}|^{2}+k_{2}\left|\nabla^{2} \tilde{w}\right|^{2}\right] d x
\end{aligned}
$$

Now, let $\gamma>0$. Application of (15) yields

$$
\begin{aligned}
\dot{\mathcal{W}}= & -\int_{\Omega}\left(c_{0}-\gamma \rho\right) \tilde{w}_{t}^{2} d x \\
& -\int_{\Omega} \sum_{j=1}^{N} H_{j} \chi_{j} \tilde{w}_{t}^{2} d x \\
& -\int_{\Gamma_{1}} c_{\Gamma}(1+\alpha) \tilde{w}_{t}^{2} d \Gamma \\
& -\int_{\Omega}\left[c_{1}\left|\nabla \tilde{w}_{t}\right|^{2}+c_{2}\left|\nabla^{2} \tilde{w}_{t}\right|^{2}\right] d x \\
& +\gamma \int_{\Omega} c_{0}\left[\left(\mu_{0} \tilde{w}\right)^{2}+\left(\frac{\tilde{w}_{t}}{\mu_{0}}\right)^{2}\right] d x \\
& +\gamma \int_{\Omega} c_{1}\left[\left(\mu_{1}|\nabla \tilde{w}|\right)^{2}+\left(\frac{\left|\nabla \tilde{w}_{t}\right|}{\mu_{1}}\right)^{2}\right] d x \\
& +\gamma \int_{\Omega} c_{2}\left[\left(\mu_{2} \nabla^{2} \tilde{w}\right)^{2}+\left(\frac{\nabla^{2} \tilde{w}_{t}}{\mu_{2}}\right)^{2}\right] d x \\
& +\gamma \int_{\Gamma_{1}} \alpha c_{\Gamma}\left[\left(\mu_{3} \tilde{w}\right)^{2}+\left(\frac{\tilde{w}_{t}}{\mu_{3}}\right)^{2}\right] d \Gamma \\
& +\gamma \int_{\Omega} \sum_{j=1}^{N} H_{j} \chi_{j}\left[\left(\mu_{4} \tilde{w}\right)^{2}+\left(\frac{\tilde{w}_{t}}{\mu_{4}}\right)^{2}\right] d x \\
& +\gamma \int_{\Gamma_{1}} c_{\Gamma}\left[\left(\mu_{5} \tilde{w}\right)^{2}+\left(\frac{\tilde{w}_{t}}{\mu_{5}}\right)^{2}\right] d \Gamma \\
& -\gamma \int_{\Omega}\left[k_{1}|\nabla \tilde{w}|^{2}+k_{2}\left|\nabla^{2} \tilde{w}\right|^{2}\right] d x, \quad \forall \mu_{i} \in \mathbb{R} \backslash\{0\} \\
& \\
& \\
& \\
& \\
&
\end{aligned}
$$

Using (10)-(11) gives

$$
\begin{aligned}
\dot{\mathcal{W}}= & -\int_{\Omega}\left[c_{0}-\gamma \rho-\frac{\gamma c_{0}}{\mu_{0}^{2}}\right] \tilde{w}_{t}^{2} d x \\
& -\int_{\Omega} \sum_{j=1}^{N} H_{j} \chi_{j}\left[1-\frac{\gamma}{\mu_{4}^{2}}\right] \tilde{w}_{t}^{2} d x \\
& -\gamma \int_{\Omega}\left[k_{1}-c_{0} \mathrm{c}_{1} \mu_{0}^{2}-c_{\Gamma} \mathrm{c}_{2}\left(\alpha \mu_{3}^{2}+\mu_{5}^{2}\right)\right. \\
& -\gamma \int_{\Omega}\left[k_{2}-c_{2} \mu_{1}^{2}-\sum_{j=1}^{N} H_{j}\left\|\chi_{j}\right\|_{\infty} \mathrm{c}_{1} \mu_{4}^{2}\right]|\nabla \tilde{w}|^{2} d x \\
& -\int_{\Omega} c_{1}\left[1-\frac{\gamma}{\mu_{1}^{2}}\right]\left|\nabla \tilde{w}_{t}\right|^{2} d x \\
& -\int_{\Omega} c_{2}\left[1-\frac{\gamma}{\mu_{2}^{2}}\right]\left|\nabla^{2} \tilde{w}_{t}\right|^{2} d x \\
& -\int_{\Gamma_{1}} c_{\Gamma}\left[1+\alpha-\frac{\gamma}{\mu_{5}^{2}}-\frac{\gamma \alpha}{\mu_{3}^{2}}\right] \tilde{w}_{t}^{2} d \Gamma
\end{aligned}
$$

for some constants $\mathrm{c}_{1}, \mathrm{c}_{2}>0$. By choosing $\mu_{0}, \ldots, \mu_{5}$ and $\gamma>0$ (such that (32) also holds) sufficiently small, there exists a constant $v>0$ such that

$$
\dot{\mathcal{W}} \leq-v \mathcal{W}, \quad t>0
$$

Thus,

$$
\mathcal{W}(t) \leq \mathcal{W}(0) e^{-v t}, \quad t \geq 0
$$


Hence, the origin $\left(\tilde{w}, \tilde{w}_{t}\right)=0$ of the observer error dynamics (23)-(27) is exponentially stable. The result is summarized in the following theorem:

Theorem 3.1 Let $H_{j}>0$ be given. The origin $\left(\tilde{w}, \tilde{w}_{t}\right)=0$ of (23)-(27) is exponentially stable.

Now, what happens if the damping coefficients $c_{1}$ and $c_{2}$ are not strictly positive? From the analysis above, it is clearly that the parameter dependent Lyapunov functional (30) is not applicable; since it depends critically on the strictly positive damping property of $\mathcal{C}$. Using the natural energy metric (31), i.e. ignoring the cross term in (30), it can be verified that the observer error dynamics (23)-(27) are stable, i.e.

$$
\dot{\mathcal{E}}=-\int_{\Omega} \tilde{w}_{t} \mathcal{C} \tilde{w}_{t} d x-\int_{\Omega} \sum_{j=1}^{N} H_{j} \tilde{w}_{t}^{2} \chi_{j} d x-\int_{\Gamma_{1}} c_{\Gamma} \tilde{w}_{t}^{2} d \Gamma
$$

The observer is thus stable, but the convergence of the observer (16)-(20) to the plant (1)-(5) can not be concluded. The inconclusive result will now be resolved by using the semigroup theory.

\subsection{Case 2: Non-strictly Positive Distributed Damping}

Let $\mathcal{C}=0$. Define $\mathbf{q}=\left(q_{1}, q_{2}\right)=\left(\tilde{w}, \tilde{w}_{t}\right)$ and the spaces

$$
\begin{aligned}
H= & H_{0}^{2}(\Omega) \times L_{2}(\Omega) \\
D(\mathbf{A})= & \left\{\mathbf{q} \in H_{0}^{4}(\Omega) \times H_{0}^{2}(\Omega)\left|\nabla^{2} q_{1}\right|_{\Gamma_{1}}=0,\right. \\
& \left.\sum_{i=1}^{2}(-1)^{i-1} \nabla^{i-1}\left(k_{i} \nabla^{i} q_{1}\right) \cdot \mathbf{n}+\left.c_{\Gamma} q_{2}\right|_{\Gamma_{1}}=0\right\}
\end{aligned}
$$

where $H_{0}^{m}(\Omega)$ and $L_{2}(\Omega)$ are given by (8) and (9), respectively. The observer error dynamics (23)-(29) can be compactly written as

$$
\frac{d}{d t} \mathbf{q}=\mathbf{A q}, \quad t>0 ; \mathbf{q}_{0} \in H
$$

where

$$
\mathbf{A q}=\left[q_{2},-\frac{1}{\rho}\left(\mathcal{K} q_{1}+\sum_{j=1}^{N} H_{j} q_{2} \chi_{j}\right)\right]^{\top}, \forall \mathbf{q} \in D(\mathbf{A})
$$

and $\mathbf{q}_{0}=\left(\tilde{w}(\cdot, 0), \tilde{w}_{t}(\cdot, 0)\right) \in H$ denotes the initial condition of the problem.

In $H$, we define the inner product

$$
\begin{aligned}
\langle\mathbf{f}, \mathbf{g}\rangle_{H}= & \int_{\Omega} \rho f_{2} g_{2} d x \\
& +\int_{\Omega}\left[k_{1} \nabla f_{1} \cdot \nabla g_{1}+k_{2} \nabla^{2} f_{1} \nabla^{2} g_{1}\right] d x
\end{aligned}
$$

where $\mathbf{f}=\left(f_{1}, f_{2}\right) \in H$ and $\mathbf{g}=\left(g_{1}, g_{2}\right) \in H$. Note that the natural energy (31) can be compactly expressed as

$$
\mathcal{E}=\frac{1}{2}\langle\mathbf{q}, \mathbf{q}\rangle_{H}=\frac{1}{2}\|\mathbf{q}\|_{H}^{2}, \quad \forall \mathbf{q} \in H
$$

It can be verified that $\left(H,\langle\cdot, \cdot\rangle_{H}\right)$ forms a Hilbert space. We have the result:

Theorem 3.2 Let $H_{j}>0$ be given. The operator $\mathbf{A}$ generates a $C_{0}$-semigroup $\left\{e^{\mathbf{A} t}\right\}_{t \geq 0}$ of contractions on $H$, and $\left\{e^{\mathbf{A} t}\right\}_{t \geq 0}$ is exponentially stable.

Proof 1 To show the first assertion, we apply the Lumer-Phillips theorem (see e.g. Pazy (1983)). It can be verified that

$$
\langle\mathbf{q}, \mathbf{A q}\rangle_{H}=-\int_{\Omega} \sum_{j=1}^{N} H_{j} \tilde{w}_{t}^{2} \chi_{j} d x-\int_{\Gamma_{1}} c_{\Gamma} \tilde{w}_{t}^{2} d \Gamma
$$

for every $\mathbf{q} \in D(\mathbf{A})$, which shows that $\mathbf{A}$ is dissipative. Consider now the equation

$$
(\lambda \mathbf{I}-\mathbf{A}) \mathbf{f}=\mathbf{g}
$$

for some given $\lambda>0$ and $\mathbf{g}=\left(g_{1}, g_{2}\right) \in H$. By the Lax-Milgram theorem (see e.g. Evans (1998)), it follows that (35) has a unique solution $\mathbf{f} \in D(\mathbf{A})$ for any given $\mathbf{g} \in H$ and $\lambda>0$. Thus, $\lambda \mathbf{I}-\mathbf{A}: H \rightarrow H$ is onto for all $\lambda>0$.

Since $\left(H,\langle\cdot, \cdot\rangle_{H}\right)$ is a Hilbert space, it follows from the argument above and (Th. 4.6, p. 16, Pazy (1983)) that $D(\mathbf{A})$ is dense in $H$, i.e. $\overline{D(\mathbf{A})}=H$. Thus, $\mathbf{A}$ generates a $C_{0}$-semigroup $\left\{e^{\mathbf{A} t}\right\}_{t \geq 0}$ of contractions on $H$.

To show the last assertion, we use a combination of the energy multipliers method and (Th. 4.1, p. 116, Pazy (1983)). Define the functional

$$
\mathcal{V}(t)=2(1-\varepsilon) t \mathcal{E}(t)+\mathcal{U}(t), \quad t \geq 0
$$

where $\varepsilon \in] 0,1[$ is an arbitrary constant, $\mathcal{E}$ is given by (31), and

$$
\mathcal{U}=2 \int_{\Omega} \mathbf{r}(x) \cdot \nabla \tilde{w} \rho \tilde{w}_{t} d x
$$

First, it can be verified that

$$
\begin{aligned}
|\mathcal{U}(t)| & \leq 2 \int_{\Omega}|\mathbf{r}(x)| \cdot|\nabla \tilde{w}| \rho\left|\tilde{w}_{t}\right| d x \\
& \leq\|\mathbf{r}\|_{\infty} \int_{\Omega} \rho\left(|\nabla \tilde{w}|^{2}+\left|\tilde{w}_{t}\right|^{2}\right) d x \\
& \leq K \mathcal{E}(t)
\end{aligned}
$$

for some constant $K>0$. Hence, the following holds

$$
[2(1-\varepsilon) t-K] \mathcal{E}(t) \leq \mathcal{V}(t) \leq[2(1-\varepsilon) t+K] \mathcal{E}(t)
$$


for $t \geq 0$.

Next, taking the time derivative of (36) along solution trajectories of (33) gives

$$
\dot{\mathcal{V}}=2(1-\varepsilon) \mathcal{E}+2(1-\varepsilon) t \dot{\mathcal{E}}+\sum_{j=1}^{4} \dot{\mathcal{U}}_{j}
$$

where $\mathcal{E}$ and $\dot{\mathcal{E}}$ are given by (31) and (34), respectively, for sufficiently large time, and

$$
\begin{aligned}
\dot{\mathcal{U}}_{1}= & 2 \int_{\Omega} \mathbf{r} \cdot \nabla \tilde{w} \nabla\left(k_{1} \nabla \tilde{w}\right) d x \\
= & \int_{\Gamma} k_{1} \mathbf{r} \cdot \mathbf{n}|\nabla \tilde{w}|^{2} d \Gamma-\int_{\Omega} k_{1} \nabla \cdot \mathbf{r}|\nabla \tilde{w}|^{2} d x \\
\dot{\mathcal{U}}_{2}= & -2 \int_{\Omega} \mathbf{r} \cdot \nabla \tilde{w} \nabla^{2}\left(k_{2} \nabla^{2} \tilde{w}\right) d x \\
= & -2 \int_{\Gamma} \mathbf{r} \cdot \nabla \tilde{w} \nabla\left(k_{2} \nabla^{2} \tilde{w}\right) \cdot \mathbf{n} d \Gamma \\
& +2 \int_{\Gamma} \mathbf{n} \cdot \nabla \tilde{w}(\nabla \cdot \mathbf{r}) k_{2} \nabla^{2} \tilde{w} d \Gamma \\
& +\int_{\Gamma} k_{2} \mathbf{r} \cdot \mathbf{n}\left|\nabla^{2} \tilde{w}\right|^{2} d \Gamma \\
& -3 \int_{\Omega} k_{2} \nabla \cdot \mathbf{r}\left|\nabla^{2} \tilde{w}\right|^{2} d x \\
\dot{\mathcal{U}}_{3}= & -2 \int_{\Omega} \mathbf{r} \cdot \nabla \tilde{w} \sum_{j=1}^{N} H_{j} \tilde{w}_{t} \chi_{j} d x \\
\leq & \sum_{j=1}^{N} 2 H_{j}\|\mathbf{r}\|_{\infty} \int_{\Omega}\left[\left(\frac{\tilde{w}_{t}}{\mu_{6}}\right)^{2}+\left(\mu_{6}|\nabla \tilde{w}|\right)^{2}\right] \chi_{j} d x \\
\dot{\mathcal{U}}_{4}= & 2 \int_{\Omega} \mathbf{r} \cdot \nabla \tilde{w}_{t} \rho \tilde{w}_{t} d x \\
= & \int_{\Gamma} \rho \mathbf{r} \cdot \mathbf{n} \tilde{w}_{t}^{2} d \Gamma-\int_{\Omega} \rho \nabla \cdot \mathbf{r} \tilde{w}_{t}^{2} d x
\end{aligned}
$$

for all $\mu_{6} \in \mathbb{R} \backslash\{0\}$, where (13)-(15) have been applied. Hence,

$$
\begin{aligned}
\dot{\mathcal{V}} \leq & -\int_{\Omega} \rho[\nabla \cdot \mathbf{r}-1+\varepsilon] \tilde{w}_{t}^{2} d x \\
& -\int_{\Omega}\left[k_{1} \nabla \cdot \mathbf{r}-(1-\varepsilon) k_{1}\right. \\
& \left.\quad-\sum_{j=1}^{N} 2 H_{j}\|\mathbf{r}\|_{\infty} \mu_{6}^{2} \chi_{j}\right]|\nabla \tilde{w}|^{2} d x \\
& -\int_{\Omega}\left[3 k_{2} \nabla \cdot \mathbf{r}-(1-\varepsilon) k_{2}\right. \\
& \left.-2 c_{\Gamma}\|\mathbf{r}\|_{\infty} c_{2} \mu_{7}^{2}\right]\left|\nabla^{2} \tilde{w}\right|^{2} d x \\
& -\int_{\Omega} \sum_{j=1}^{N} 2 H_{j} \chi_{j}\left[(1-\varepsilon) t-\frac{\left.\|\mathbf{r}\|_{\infty}\right] \tilde{w}_{t}^{2} d x}{\mu_{6}^{2}}\right. \\
& -\int_{\Gamma_{1}}\left[2(1-\varepsilon) c_{\Gamma} t-\rho \mathbf{r} \cdot \mathbf{n}-2 c_{\Gamma} \frac{\|\mathbf{r}\|_{\infty}}{\mu_{7}^{2}}\right] \tilde{w}_{t}^{2} d \Gamma \\
& -\int_{\Gamma_{1}} k_{1} \mathbf{r} \cdot \mathbf{n}|\nabla \tilde{w}|^{2} d \Gamma-\int_{\Gamma_{0}} k_{2}|\mathbf{r} \cdot \mathbf{n}|\left|\nabla^{2} \tilde{w}\right|^{2} d \Gamma
\end{aligned}
$$

for $t>0$ and $\forall \mu_{7} \in \mathbb{R} \backslash\{0\}$, where (11), (24)-(27) and the assumption $\boldsymbol{A} .2$ have been applied. Note that $\nabla \cdot \mathbf{r} \geq 1,\left.\mathbf{r} \cdot \mathbf{n}\right|_{\Gamma_{0}} \leq 0$ and $\left.\mathbf{r} \cdot \mathbf{n}\right|_{\Gamma_{1}}>0$.

Now, let $\varepsilon \in] 0,1\left[\right.$ be fixed and choose $\mu_{6}, \mu_{7}$ sufficiently small. Thus, the following holds

$$
\dot{\mathcal{V}}(t) \leq 0, \quad t \geq t_{1}
$$

$$
t_{1}=\max \left\{\frac{\|\mathbf{r}\|_{\infty}}{(1-\varepsilon) \mu_{6}^{2}}, \frac{\|\mathbf{r}\|_{\infty}\left(\rho+\frac{2 c_{\Gamma}}{\mu_{7}^{2}}\right)}{2(1-\varepsilon) c_{\Gamma}}\right\}
$$

Moreover, by (34) and (38)-(39), we have

$$
\mathcal{E}(t) \leq \frac{K}{2(1-\varepsilon) t-K} \mathcal{E}(0), \quad t \geq t_{\max }
$$

where

$$
t_{\max }=\max \left\{t_{1}, \frac{K}{2(1-\varepsilon)}\right\}
$$

Since $\mathcal{E}(t)=\frac{1}{2}\|\mathbf{q}(t)\|_{H}^{2}$, it follows that $\|\mathbf{q}(t)\|_{H}<\infty$, $\forall t \geq 0$, and decays as $O(1 / \sqrt{t})$ for sufficiently large time. Thus,

$$
\int_{0}^{\infty}\|\mathbf{q}(t)\|_{H}^{2 q} d t=\int_{0}^{\infty}\left\|e^{\mathbf{A} t} \mathbf{q}_{0}\right\|_{H}^{2 q} d t<\infty
$$

$\forall q>1$ and $\forall \mathbf{q}_{0} \in D(\mathbf{A})$. By density of $D(\mathbf{A})$ in $H$, the following also holds

$$
\int_{0}^{\infty}\|\mathbf{q}(t)\|_{H}^{2 q} d t<\infty
$$

$\forall q>1$ and $\forall \mathbf{q}_{0} \in H$. According to (Th. 4.1, p. 116, Pazy (1983)), there exist constants $M \geq 1$ and $\kappa>0$ such that

$$
\left\|e^{\mathbf{A} t}\right\|_{H} \leq M e^{-\kappa t}, \quad t \geq 0
$$

i.e.

$$
\|\mathbf{q}(t)\|_{H} \leq M e^{-\kappa t}\|\mathbf{q}(0)\|_{H}, \quad t \geq 0
$$

$\forall \mathbf{q}(0) \in H$.

Remark 3.1 Note that the stability analysis of the observer (16)-(20) is based on the vector first order form, i.e. (33). But the design and implementation of the observers are based on the original form of the system, i.e. (1)-(5). Hence, the advantageous algebraic structures of the system are preserved.

Remark 3.2 Let the control law $u$ be designed such that the closed loop system (1)-(7) is wellposed, i.e. the closed loop system of (1)-(7) has a unique solution. Since $\tilde{w}(\mathbf{x}, t)=\hat{w}(\mathbf{x}, t)-w(\mathbf{x}, t)$, it follows from Theorem 2 that the observer (16)-(22) is wellposed. 


\section{Conclusions}

Observer design for second-order distributed parameter systems in $\mathbb{R}^{2}$ is studied. Based on finite number of measurements, exponentially stable observer is designed. The existence, uniqueness and stability of the observer are based on semigroup theory.

\section{References}

Anderson, B. D. O. and Moore, J. B. Optimal Control: Linear Quadratic Methods. Prentice-Hall, 1990.

Balas, M. J. Do all linear flexible structures have convergent second-order observers? AIAA Guidance, Control, Dynamics, 1999. Vol. 22, No. 6.

Bounit, H. and Hammouri, H. Observers for infinite dimensional bilinear systems. Euro. J. Control, 1997. Vol. 2.

Demetriou, M. A. Second order observers for second order distributed parameter systems. Systems Control Letters, 2004. Vol. 51.

Evans, L. C. Partial differential equations. American Mathematical Society, 1998. Vol. 19.

Gauthier, J.-P. and Kupka, I. Deterministic observation theory and applications. Cambridge University Press, 2001.

I., S. and Dym, C. Energy and Finite Element Methods in Structural Mechanics. Hemishere Publishing Corporation, 1991.

Kristiansen, D. Modeling of Cylinder Gyroscopes and Observer Design for Nonlinear Oscillations. Ph.D. thesis, Norwegian University of Science and Technology, Trondheim, Norway, 2000.

Luenberger, D. L. Introduction to Dynamic Systems. Wiley, 1979.

Nguyen, T. D. and Egeland, O. Tracking and observer design for a motorized euler-bernoulli beam. Proc. IEEE Int. Conference on Decision and Control, Maui, Hawaii, 2003.

Nguyen, T. D. and Egeland, O. Second-order observer for a class of second-order distributed parameter systems. Proc. IEEE Int. Conference on Decision and Control, San Diego, CA, 2006.

Nijmeijer, H. and (eds.), T. I. F. New Directions in Nonlinear Observer Design. Springer-Verlag, 1999.
Pazy, A. Semigroups of Linear Operators and Applications to Partial Differential Equations. SpringerVerlag, 1983.

Smyshlyaev, A. and Kristic, M. Backstepping observers for a class of parabolic pdes. Systems and Control Letters, 2005. Vol. 54.

Vazquez, R. and Krstic, M. A close-form observer for the channel flow navier-stokes system. Proc. IEEE Int. Conference on Decision and Control, Seville, Spain, 2005.

Xu, D. J., C.-Z. and Sallet, G. Infinite dimensional observers for vibrating systems. Proc. IEEE Int. Conference on Decision and Control, San Diego, CA, 2006. 